\title{
Reconciling conflicting electrophysiological findings on the guidance of attention by working memory
}

\author{
Nancy B. Carlisle • Geoffrey F. Woodman
}

Published online: 6 August 2013

(C) Psychonomic Society, Inc. 2013

\begin{abstract}
Maintaining a representation in working memory has been proposed to be sufficient for the execution of topdown attentional control. Two recent electrophysiological studies that recorded event-related potentials (ERPs) during similar paradigms have tested this proposal, but have reported contradictory findings. The goal of the present study was to reconcile these previous reports. To this end, we used the stimuli from one study (Kumar, Soto, \& Humphreys, 2009) combined with the task manipulations from the other (Carlisle \& Woodman, 2011b). We found that when an item matching a working memory representation was presented in a visual search array, we could use ERPs to quantify the size of the covert attention effect. When the working memory matches were consistently task-irrelevant, we observed a weak attentional bias to these items. However, when the same item indicated the location of the search target, we found that the covert attention effect was approximately four times larger. This shows that simply maintaining a representation in working memory is not equivalent to having a top-down attentional set for that item. Our findings indicate that high-level goals mediate the relationship between the contents of working memory and perceptual attention.
\end{abstract}

Keywords Object-based attention · Working memory · Electrophysiology

N. B. Carlisle $(\triangle)$

Center for Mind and Brain, University of California,

Davis, CA, USA

e-mail: nancy.carlisle@gmail.com

G. F. Woodman

Vanderbilt Center for Cognitive and Integrative Neuroscience,

Vanderbilt Vision Research Center, Department of Psychology,

Vanderbilt University, Nashville, TN, USA

e-mail: geoffrey.f.woodman@vanderbilt.edu
Top-down control is critical in essentially every theory of attention (Bundesen, 1990; Desimone \& Duncan, 1995; Duncan \& Humphreys, 1989; Wolfe, Cave, \& Franzel, 1989). Top-down control directs the mechanisms of perceptual attention to relevant items in the visual field, interacting with bottom-up factors, such as stimulus salience, to determine what stimuli are processed most efficiently. The biased-competition theory of attention (Desimone \& Duncan, 1995) specifies a direct connection between the contents of working memory (WM) and top-down attention. According to biased competition, maintaining target information in WM leads to elevated activity in the cells selective for target features. This creates a competitive advantage for target-matching items in the visual field, increasing the probability that task-relevant information will be represented. This elegantly simple mechanism suggests a mandatory link between WM storage and top-down attentional control.

Conflicting findings in studies that have focused on whether WM maintenance is sufficient to control attention have spurred a growing debate (for reviews, see Olivers, Peters, Houtkamp, \& Roelfsema, 2011; Soto, Hodsoll, Rotshtein, \& Humphreys, 2008). In these studies, participants' WM is typically loaded, and then they perform a visual search task that occasionally includes a memory-matching distractor. If WM maintenance is sufficient to provide top-down attentional control, attention should involuntarily be guided to WM-matching items in the visual field. Many studies have reported that attention is drawn to WM-matching distractors, which is most frequently evidenced by increased search reaction times (RTs) when a WM-matching distractor is present in the array (Olivers, Meijer, \& Theeuwes, 2006; Soto, Heinke, Humphreys, \& Blanco, 2005; Soto \& Humphreys, 2007, 2009; Soto, Humphreys, \& Heinke, 2006). However, notable exceptions (Dalvit \& Eimer, 2011; Dombrowe, Olivers, \& Donk, 2010; Houtkamp \& Roelfsema, 2006), including reports of faster RTs when WMmatching distractors are present (Downing \& Dodds, 2004; Han \& Kim, 2009; Woodman \& Luck, 2007), suggest that 
task-irrelevant WM matches do not always compete for attention and can be effectively ignored (e.g., Arita, Carlisle, \& Woodman, 2013).

Due to the contradictory findings from behavioral measures, converging evidence from event-related potentials (ERPs) has been sought in order to provide definitive tests of WM's guidance of attention (Carlisle \& Woodman, 2011b; Kumar, Soto, \& Humphreys, 2009; Peters, Goebel, \& Roelfsema, 2009). The temporal resolution of ERPs can show early differences in attentional selection that might not be evident in behavioral output. However, two previous ERP studies with similar tasks have reported contradictory results. Kumar, Soto, and Humphreys loaded WM with a colored shape (Fig. 1A) that could be present as a distractor in a subsequent search task for a tilted cyan line. The N2pc component was measured to determine whether the WM matches captured attention. The N2pc (for N2-posterior-contralateral) can track the focus of covert attention (Woodman \& Luck, 1999, 2003), with the electrodes contralateral to the focus of perceptual attention becoming more negative than ipsilateral electrodes (Woodman \& Luck, 2003). When the memorymatching distractor was in the opposite hemifield from the search target, Kumar and colleagues reported that targets elicited a reduced-amplitude N2pc relative to target-only trials, whereas trials with a memory-matching distractor in the same hemifield as the target elicited an increased-amplitude $\mathrm{N} 2 \mathrm{pc}$ relative to target-only trials. This pattern was taken as evidence that the WM matches captured attention, leading to the conclusion that maintaining a representation in WM is sufficient to create top-down control of attention.

As is shown in Fig. 1B, Carlisle and Woodman (2011b) used a highly similar paradigm. However, they reported a very different pattern of results. Across three experiments in which they manipulated features of the search display, they found no evidence of an N2pc to the memory-matching distractors. Instead, they found evidence that memory-matching items were suppressed, because they elicited a lateralized positivity
(Hickey, Di Lollo, \& McDonald, 2009). In Experiment 4, the memory-matching item was the search target. When the memory match was task-relevant because it indicated the search target, it elicited a large N2pc, demonstrating that the stimuli were capable of producing early and robust N2pc effects. Overall, this suggested that goals were necessary for creating a top-down attentional bias from the memory representation.

The conflicting findings and conclusions from the Kumar et al. (2009) and Carlisle and Woodman (2011b) studies, as well as from previous behavioral work, have done little to resolve the debate in the literature about the mechanism of top-down attentional control. Throughout this debate, different laboratories have consistently used different stimuli, with few exceptions (Olivers, 2009). Here, we sought to reach across this divide by using the stimuli of Kumar et al., while manipulating the goal relevance of the memory-matching items across experiments, as in Carlisle and Woodman (2011b). Specifically, we felt it necessary to use the stimuli from the Kumar et al. study because they consisted of large colored shapes, one of which could match the memory representation, with a small tilted line as the search target element. This difference in the saliency between the memory-matching information and the search target might have contributed importantly to the ERP and behavioral effects observed. We then manipulated the goal relevance of the memory-matching items for search, as in Carlisle and Woodman's (2011b) study. The memory-matching items never contained the search target element in Experiment 1A, whereas the memory-matching items always contained the search target element in Experiment $1 \mathrm{~B}$. This allowed us to determine whether the strengths of the attentional bias to the salient memory-matching items of Kumar et al. were always similar, regardless of goal of the search task at hand.

If a match between the bottom-up input and the memory representation is sufficient to control attention with the Kumar stimuli, we would expect to find similar N2pcs to the salient memory-matching items, regardless of their task relevance
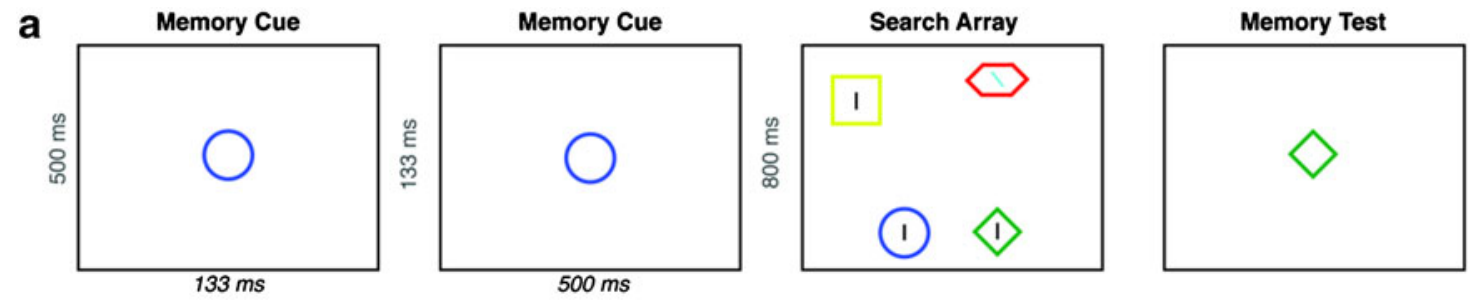

b
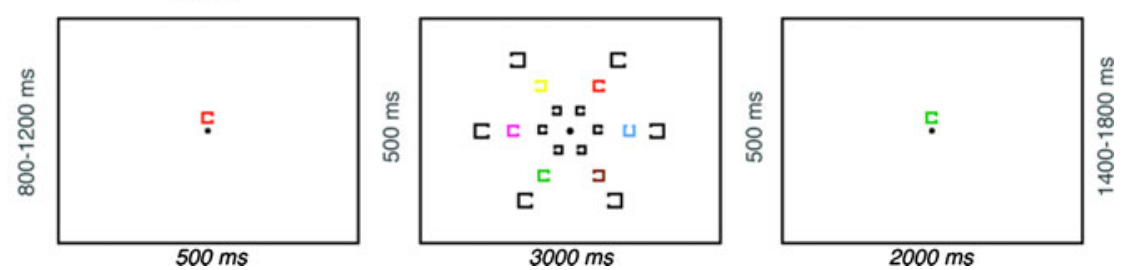

Fig. 1 (A) Stimulus sequences from Kumar, Soto, and Humphreys (2009). (B) Stimulus sequences from Carlisle and Woodman (2011b). The stimuli are not to scale, but represent the overall stimulus differences. Note that in the present work, a fixation point was added to the stimuli of Kumar et al 
(i.e., the same N2pc to memory matches across Exp. 1A, in which the match was irrelevant for search, and Exp. 1B, it which it was relevant for search). In contrast, if the control of attention by WM is dependent on goals, we would expect to find a larger N2pc for the task-relevant memory matches in Experiment $1 \mathrm{~B}$, relative to the task-irrelevant memory matches in Experiment 1A.

\section{Method}

\section{Participants}

A group of 13 individuals from the Vanderbilt community participated in these experiments. All gave informed consent and were compensated at a rate of $\$ 10 / \mathrm{h}$. Participants reported no history of neurological problems, normal or corrected-tonormal visual acuity, and normal color vision, and were between the ages of 18 and 35.

\section{Stimuli and procedure}

In Experiment 1A, participants performed the task of Kumar et al. (2009) (see Fig. 1A). The memory and search items were combinations of five possible colors (red, yellow, blue, green, or pink) and five possible outlined shapes (a circle, subtending $1.80^{\circ} \times 1.80^{\circ}$ of visual angle; hexagon, $2.38^{\circ} \times 0.95^{\circ}$; square, $1.50^{\circ} \times 1.50^{\circ}$; diamond, $1.91^{\circ} \times 1.91^{\circ}$; or triangle, $2.00^{\circ} \times$ $1.50^{\circ}$, with each line width $0.24^{\circ}$ ). The memory cue and test arrays contained one item centered on the screen. The search arrays contained four objects presented on an imaginary circle $6^{\circ}$ from the screen center, with one object per quadrant either $30^{\circ}$ or $60^{\circ}$ from vertical. The search target was a cyan line $\left(0.57^{\circ} \times 0.12^{\circ}\right.$, tilted $38^{\circ}$ from vertical $)$ centered in one of the objects. All other objects had a black vertical line $\left(0.57^{\circ} \times\right.$ $0.12^{\circ}$ ) presented in their center. A memory-matching distractor was present on half of the search trials. We only deviated from the methods of Kumar et al. in that we added a black fixation point $\left(0.18^{\circ} \times 0.18^{\circ}\right)$ beginning $800-1,200 \mathrm{~ms}$ before the memory cue until the end of the trial. This was necessary in order to help participants maintain fixation throughout each trial. Otherwise, all stimulus and timing parameters were identical to those of Kumar et al.

Participants were first shown an object to keep in memory (133 ms on, $133 \mathrm{~ms}$ off, and $500 \mathrm{~ms}$ on, followed by a $800-\mathrm{ms}$ fixation-only period). Participants were then presented with the search array $(2,500 \mathrm{~ms})$ and reported whether the top of the cyan line tilted to the left or the right of vertical by pressing a left or right directional arrow on a gamepad with their left hand. On $20 \%$ of trials, a memory test was presented after the search array (800-ms blank screen followed by 2,000-ms memory test), with change and no-change tests being equally likely. Participants reported "change" or "no change" by pressing one of two buttons on the gamepad with their right hand. They were explicitly instructed that the search target line would never be within the memory match. After a short practice, they completed 400 experimental trials.

In Experiment 1B, all of the stimuli and procedures were the same, except as follows. The memory-matching item was now present on each trial and contained a black line tilted $38^{\circ}$ to the left or the right of vertical, which served as the search target. Participants' task was to report the tilt of this black line, and thus the tilted cyan line in one of the other colored shapes was now a distractor. Participants were informed that the black tilted line would always appear on the memory-matching item.

\section{ERP recording and analysis}

The electroencephalogram (EEG) was recorded from electrodes in an elastic cap (Electrocap International) using a subset of the International 10-20 sites (Fz, Cz, Pz, F3/F4, $\mathrm{C} 3 / \mathrm{C} 4, \mathrm{P} 3 / \mathrm{P} 4, \mathrm{PO} 3 / \mathrm{PO} 4, \mathrm{~T} 3 / \mathrm{T} 4, \mathrm{~T} 5 / \mathrm{T} 6$, and O1/O2) and sites $\mathrm{OL} / \mathrm{OR}$ (between $\mathrm{O} 1$ and $\mathrm{T} 5$ and $\mathrm{O} 2$ and $\mathrm{T} 6$, respectively) using our standard methods (i.e., Carlisle, Arita, Pardo, \& Woodman, 2011; Carlisle \& Woodman, 2011b). Trials containing ocular or myogenic artifacts were excluded from the averages (see Woodman \& Luck, 2003). Participants with artifacts on more than $25 \%$ of correct search trials or residual systematic eye movements greater than $3.2 \mu \mathrm{V}$ after averaging would have been replaced, but no participants met these criteria (one participant did not complete the study due to being unable to maintain fixation, and was replaced). Averages of $10.3 \%$ and $9.3 \%$ of trials per participant were rejected in Experiments $1 \mathrm{~A}$ and $1 \mathrm{~B}$, respectively.

We measured the N2pc amplitude to the target as the difference in mean amplitudes from 200 to $300 \mathrm{~ms}$ after the search onset between electrode sites O1/2, OL/R, and T5/6 contralateral versus ipsilateral to the target location on correct search trials. Similarly, the N2pc to the memory-matching item was the difference between electrodes contralateral versus ipsilateral to the memory-match location. In Experiment 1B, the memory-match location was also the search target location.

The fractional-area latency method was used to measure the onset of the $\mathrm{N} 2 \mathrm{pc}$ in order to provide an empirical metric of the salience of the memory-matching item relative to the tilted-line search target (Luck, 2005; Woodman, 2010). The $\mathrm{N} 2 \mathrm{pc}$ onset was measured as the time point at which $25 \%$ of the area under the curve had occurred for each participant's averaged contralateral-minus-ipsilateral waveforms, relative to the memory match. The measurement window for the $\mathrm{N} 2 \mathrm{pc}$ was set to match the window used in all of the other $\mathrm{N} 2 \mathrm{pc}$ analyses, resulting in a window that spanned 200$300 \mathrm{~ms}$ poststimulus. Only trials on which no memory item was present were included in the analysis of the tilted-line search target, to remove any influence of the memorymatching distractor on our fractional area latency measures. 
Greenhouse-Geisser epsilon corrections for nonsphericity (Jennings \& Wood, 1976) were applied to the $p$ values when appropriate, for all analyses.

\section{Results}

In Experiment 1A, participants were $99 \%$ correct on the search task, and responses were faster when the memory match was absent $(727 \mathrm{~ms})$ than when it was present $(764$ $\mathrm{ms}), t(12)=4.51, p<.001$. On the $20 \%$ of trials that contained a memory test, participants were $94 \%$ correct on trials (with $3 \%$ of the trials being incorrect due to no response). We found no significant difference based on whether the memory match had been present versus absent from the search array $(95 \%$ vs. $94 \%$ correct, $p=.33$ )

Figure 2A shows the N2pcs to the search target. The amplitudes of the target $\mathrm{N} 2 \mathrm{pc}$ were similar when no memorymatching item was present $(0.83 \mu \mathrm{V})$ and when the memorymatching item was in the same hemifield as the search target $(0.82 \mu \mathrm{V})$, and smaller when the memory-matching item was in the opposite hemifield $(0.15 \mu \mathrm{V})$. We performed an analysis of variance (ANOVA) with the factors Contralaterality (ipsilateral vs. contralateral to the target), Array Type (no memory vs. memory same hemifield as target vs. memory opposite hemifield from target), and Electrode Site (O1/2 vs. OL/R vs. T5/6). This showed significant main effects of contralaterality $[F(1,12)=11.33, M S E=1.86, p<.01]$, indicating that the N2pc to the search target was significant, and electrode $[F(2,24)=$
4.75, MSE $=13.75, p<.05]$, due to generally more positive waveforms at $\mathrm{OL} / \mathrm{R}$, followed by $\mathrm{T} 5 / 6$ and then $\mathrm{O} 1 / 2$. We found an interaction of contralaterality and electrode $[F(2,24)=4.57$, $M S E=0.08, p<.05]$, with a larger $\mathrm{N} 2 \mathrm{pc}$ at $\mathrm{OL} / \mathrm{R}$ than at $\mathrm{O} 1 / 2$ and T5/6. Critically, a significant Array Type $\times$ Contralaterality interaction emerged $[F(2,24)=5.57, M S E=0.54, p<.05]$. Follow-up analyses showed that this interaction was driven by the N2pc to the search target in the opposite hemifield from the memory match being significantly smaller than when the memory match was in the same hemifield $[t(12)=2.39, p<.05]$ or when no memory match was present $[t(12)=3.29, p<.01]$. No other main effects or interactions were significant.

Next, we examined the N2pc to the memory-matching item. Figure $2 \mathrm{~B}$ shows that the waveforms contralateral to the memory match were more negative than the ipsilateral waveforms (i.e., a $0.34-\mu \mathrm{V}$ difference). We performed an ANOVA with the factors Contralaterality (ipsilateral vs. contralateral to the memory match) and Electrode Site (O1/2 vs. OL/R vs. T5/6). We found significant effects of electrode site $[F(2,24)=4.55, M S E$ $=4.73, p<.05]$ and contralaterality $[F(1,12)=5.82, M S E=$ $0.38, p<.05]$, indicating that participants were attending to the memory-matching distractor.

In Experiment 1B, participants were highly accurate on the search task (99\% correct) and the memory task (96\% correct, including no response recorded on $3 \%$ of the trials). As is shown in Fig. 2C, the task-relevant memory-matching item elicited a large N2pc (i.e., $1.30 \mu \mathrm{V}$ ). An ANOVA with the factors Contralaterality and Electrode showed significant effects of contralaterality $[F(1,12)=31.84, M S E=1.03, p<.001]$ and

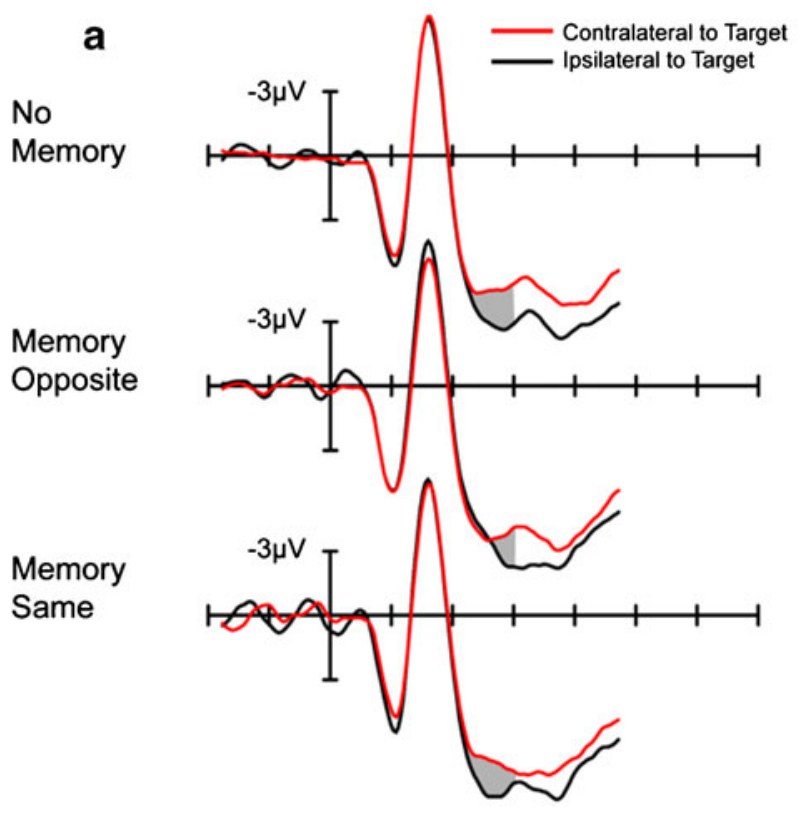

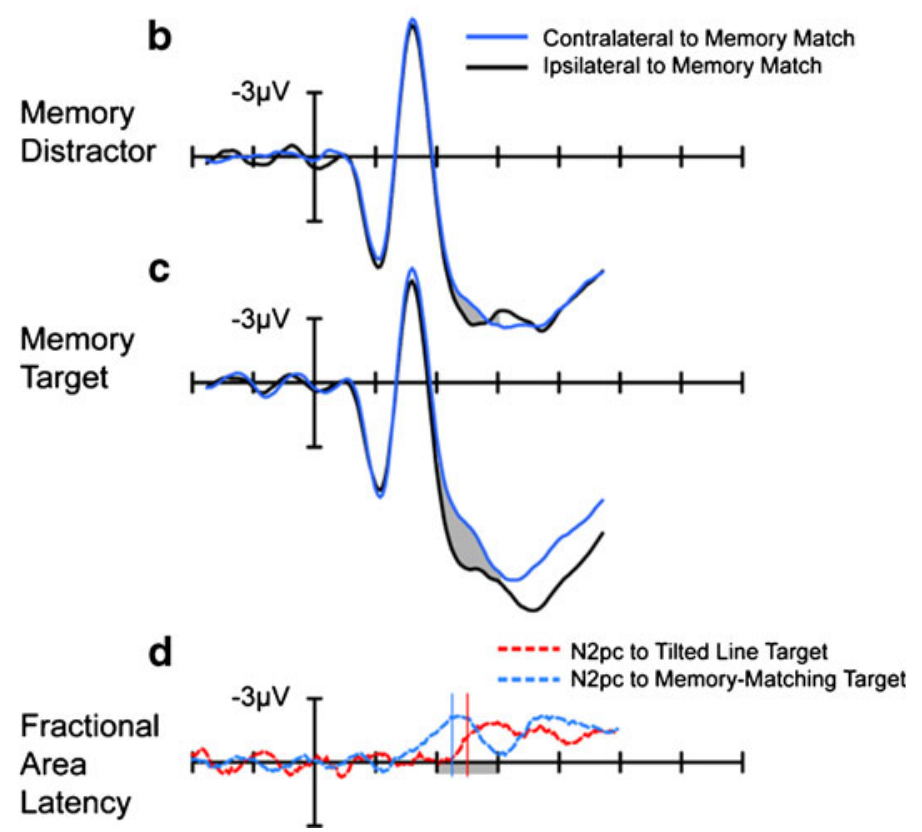

1B. (D) N2pc difference waves (contralateral - ipsilateral) to the tiltedline target in Experiment $1 \mathrm{~A}$ and the memory-matching target in Experiment 1B. Vertical lines indicate the points of $25 \%$ fractional-area latency of the N2pcs from 200 to $300 \mathrm{~ms}$ after search array onset
Fig. 2 Event-related potentials (ERPs) recorded at electrodes OL/R. (A) N2pcs to the search target, as a function of the array type in Experiment 1A. (B) N2pc to the task-irrelevant memory-matching distractor in Experiment 1A. (C) N2pc to the task-relevant memory match in Experiment 
electrode site $[F(2,24)=8.47, M S E=3.85, p<.05]$, as well as a Contralaterality $\times$ Electrode interaction $[F(2,24)=9.46, M S E=$ $0.05, p<.01]$, due to $\mathrm{N} 2 \mathrm{pc}$ amplitudes being largest at $\mathrm{OL} / \mathrm{R}$, followed by T5/6 and then O1/2. A planned comparison of the N2pcs elicited by memory matches in Experiment $1 \mathrm{~A}$ relative to Experiment 1B confirmed that the N2pc to the memory matches was significantly larger when the match was taskrelevant rather than task-irrelevant $[t(12)=4.08, p<.01]$.

Finally, we performed a $25 \%$ fractional-area latency measure to contrast the speeds of orienting to the tilted-line target in Experiment 1A and the memory-matching search target in Experiment 1B. As is shown in Fig. 2D, the 25\% fractionalarea latency occurred at $250 \mathrm{~ms}$ when participants were orienting to the tilted-line search target and at $228 \mathrm{~ms}$ when participants were orienting to the memory-matching search target. A paired-samples $t$ test indicated that the onset of the N2pc was significantly earlier in Experiment $1 \mathrm{~B}$ than in Experiment $1 \mathrm{~A}[t(12)=3.84, p<.01]$.

\section{Discussion}

The memory-matching distractors did significantly modulate the amplitude of the N2pc to the search target, replicating the pattern reported by Kumar et al. (2009). In addition, the significant N2pc elicited by the memory match throughout Experiment 1 showed that the memory-matching item was attended even when it was a distractor. In Experiment 1B, the taskrelevant memory matches elicited a robust N2pc, approaching 4 times larger than the N2pc to the memory-matching distractor in Experiment 1A. ${ }^{1}$ This pattern replicated the goal-dependent influence of WM on the deployment of attention, as measured using the N2pcs described in Carlisle and Woodman (2011b). As we will discuss next, this does not mean that the bottom-up characteristics of the stimuli were not important. Indeed, the present finding of a significant N2pc to memory-matching items indicates that a highly salient memory-matching input may be necessary in order to observe attentional biases to such items.

Our findings provide a reconciliation of seemingly contradictory findings in the literature. It appears that the stimuli used in the study of Kumar et al. (2009) and in most of the experiments from their group (e.g., Soto et al., 2005; 2008) do bias attention to memory-matching items more strongly than do the stimuli in Carlisle and Woodman (2011b). This is not surprising when we consider the physical characteristics of these stimuli. The memory and search stimuli used here and in Kumar et al.'s study consisted of a larger memory-matching

\footnotetext{
${ }^{1}$ Due to a lack of clarity on our part regarding the precise stimuli used in Kumar et al. (2009), we observed this same general pattern of results in a previous study when we used filled colored shapes. Specifically, we found that the amplitude of the N2pc to a memory-matching distractor in the search array was approximately 3.9 times smaller than when that item contained the search target line.
}

item (i.e., a large colored shape outline) and a less salient target feature (i.e., a small, oriented line). Our fractional-area latency measures confirmed that participants could orient more quickly to the memory item in Experiment 1B than to the oriented line target in Experiment 1A. In Carlisle and Woodman (2011b) and in many of the previous reports in which memorymatching items had no effect, or even facilitated visual search performance - the features that defined the search target and the memory match were similarly salient (the same size, shape, average luminance, etc.; see Downing \& Dodds, 2004; Peters et al., 2009; Woodman \& Luck, 2007). The present study demonstrates that a highly salient memory-matching input will have a stronger influence on attention than stimuli that are matched for saliency (see also Olivers, 2009).

In addition to suggesting that the physical stimuli matter, the present study shows that goals matter, too. That is, even with a memory-matching stimulus that was much more salient than the search target, the task relevance of the stimuli modulated the size of the attention effects approximately fourfold. This shows that simply representing a target in WM is not sufficient to control attention at the level of a search template. Instead, top-down control is contingent on higher-level control settings in the brain that can make use of the information in WM when it is consistent with the goals of the task.

The present findings are in line with a growing body of evidence that the relationship between WM and attentional guidance is flexible and dependent on the current task goals (Carlisle \& Woodman, 2011a; Han \& Kim, 2009; Moher, Abrams, Egeth, Yantis, \& Stuphorn, 2011; Peters et al., 2009). The present findings are also consistent with recent behavioral work showing that representations maintained in WM create a weaker attentional influence than does maintaining an attentional set for an item in WM (Olivers \& Eimer, 2011). Unfortunately, it seems that the elegantly simple mechanism of topdown control proposed by biased competition (Desimone \& Duncan, 1995) cannot explain the variable relationship between $\mathrm{WM}$ and attention present in the empirical literature. WM representations can be used to guide attention, but WM maintenance alone does not lead to the same attentional influence during the processing of complex visual scenes as does a goalrelated attentional template.

Author note This study was supported by the National Science Foundation (Grant No. BCS 09-57072) and by the National Eye Institute of NIH (Grant No. RO1-EY019882).

\section{References}

Arita, J. T., Carlisle, N. B., \& Woodman, G. F. (2013). Templates for rejection: Configuring attention to ignore task-irrelevant features. Journal of Experimental Psychology: Human Perception and Performance, 38, 580-584. doi:10.1037/a0027885 
Bundesen, C. (1990). A theory of visual attention. Psychological Review, 97, 523-547. doi:10.1037/0033-295X.97.4.523

Carlisle, N. B., Arita, J. T., Pardo, D., \& Woodman, G. F. (2011). Attentional templates in visual working memory. Journal of Neuroscience, 31, 9315-9322.

Carlisle, N. B., \& Woodman, G. F. (2011a). Automatic and strategic effects in the guidance of attention by working memory representations. Acta Psychologica, 137, 217-225. doi:10.1016/j.actpsy.2010. 06.012

Carlisle, N. B., \& Woodman, G. F. (2011b). When memory is not enough: Electrophysiological evidence for goal-dependent use of working memory representations in guiding visual attention. Journal of Cognitive Neuroscience, 23, 2650-2664. doi:10.1162/jocn.2011.21602

Dalvit, S., \& Eimer, M. (2011). Memory-driven attentional capture is modulated by temporal task demands. Visual Cognition, 19, 145-153.

Desimone, R., \& Duncan, J. (1995). Neural mechanisms of selective visual attention. Annual Review of Neuroscience, 18, 193-222. doi:10.1146/annurev.ne.18.030195.001205

Dombrowe, I., Olivers, C., \& Donk, M. (2010). The time course of working memory effects on visual attention. Visual Cognition, 18, 1089-1112

Downing, P., \& Dodds, C. (2004). Competition in visual working memory for control of search. Visual Cognition, 11, 689-703.

Duncan, J., \& Humphreys, G. W. (1989). Visual search and stimulus similarity. Psychological Review, 96, 433-458. doi:10.1037/0033295X.96.3.433

Han, S. W., \& Kim, M.-S. (2009). Do the contents of working memory capture attention? Yes, but cognitive control matters. Journal of Experimental Psychology: Human Perception and Performance, 35, 1292-1302. doi:10.1037/a0016452

Hickey, C., Di Lollo, V., \& McDonald, J. J. (2009). Electrophysiological indices of target and distractor processing in visual search. Journal of Cognitive Neuroscience, 21, 760-775. doi:10.1162/jocn.2009. 21039

Houtkamp, R., \& Roelfsema, P. R. (2006). The effect of items in working memory on the deployment of attention and the eyes during visual search. Journal of Experimental Psychology: Human Perception and Performance, 32, 423-442. doi:10.1037/0096-1523.32.2.423

Jennings, J. R., \& Wood, C. C. (1976). The e-adjustment procedure for repeated-measures analyses of variance. Psychophysiology, 13, 277-278.

Kumar, S., Soto, D., \& Humphreys, G. W. (2009). Electrophysiological evidence for attentional guidance by the contents of working memory. European Journal of Neuroscience, 30, 307-317.

Luck, S. J. (2005). An introduction to the event-related potential technique. Cambridge, MA: MIT Press.

Moher, J., Abrams, J., Egeth, H. E., Yantis, S., \& Stuphorn, V. (2011). Trial-by-trial adjustments of top-down set modulate oculomotor capture. Psychonomic Bulletin \& Review, 18, 897-903. doi:10. 3758/s13423-011-0118-5

Olivers, C. N. L. (2009). What drives memory-driven attentional capture? The effects of memory type, display type, and search type. Journal of Experimental Psychology: Human Perception and Performance, 35, 1275-1291. doi:10.1037/a0013896

Olivers, C. N. L., \& Eimer, M. (2011). On the difference between working memory and attentional set. Neuropsychologia, 49, 15531558. doi:10.1016/j.neuropsychologia.2010.11.033

Olivers, C. N. L., Meijer, F., \& Theeuwes, J. (2006). Feature-based memory-driven attentional capture: Visual working memory content affects visual attention. Journal of Experimental Psychology: Human Perception and Performance, 32, 1243-1265. doi:10.1037/ 0096-1523.32.5.1243

Olivers, C. N. L., Peters, J., Houtkamp, R., \& Roelfsema, P. R. (2011). Different states in visual working memory: When it guides attention and when it does not. Trends in Cognitive Sciences, 15, 327-334. doi:10.1016/j.tics.2011.05.004

Peters, J. C., Goebel, R., \& Roelfsema, P. R. (2009). Remembered but unused: The accessory items in working memory that do not guide attention. Journal of Cognitive Neuroscience, 21, 1081-1091.

Soto, D., Heinke, D., Humphreys, G. W., \& Blanco, M. J. (2005). Early, involuntary top-down guidance of attention from working memory. Journal of Experimental Psychology: Human Perception and Performance, 31, 248-261. doi:10.1037/0096-1523.31.2.248

Soto, D., Hodsoll, J. P., Rotshtein, P., \& Humphreys, G. W. (2008). Automatic guidance of attention from working memory. Trends in Cognitive Sciences, 12, 342-348. doi:10.1016/j.tics.2008.05.007

Soto, D., \& Humphreys, G. W. (2007). Automatic guidance of visual attention from verbal working memory. Journal of Experimental Psychology: Human Perception and Performance, 33, 730-737. doi:10.1037/0096-1523.33.3.730

Soto, D., \& Humphreys, G. W. (2009). Automatic selection of irrelevant object features through working memory. Experimental Psychology, 56, 165-172.

Soto, D., Humphreys, G. W., \& Heinke, D. (2006). Working memory can guide pop-out search. Vision Research, 46, 1010-1018. doi:10. 1016/j.visres.2005.09.008

Wolfe, J. M., Cave, K. R., \& Franzel, S. L. (1989). Guided search: An alternative to the feature integration model for visual search. Journal of Experimental Psychology: Human Perception and Performance, 15, 419-433. doi:10.1037/0096-1523.15.3.419

Woodman, G. F. (2010). A brief introduction to the use of event-related potentials in studies of perception and attention. Attention, Perception, \& Psychophysics, 72, 2031-2046. doi:10.3758/APP.72.8.2031

Woodman, G. F., \& Luck, S. J. (1999). Electrophysiological measurement of rapid shifts of attention during visual search. Nature, 400, 867-869.

Woodman, G. F., \& Luck, S. J. (2003). Serial deployment of attention during visual search. Journal of Experimental Psychology: Human Perception and Performance, 29, 121-138. doi:10.1037/00961523.29.1.121

Woodman, G. F., \& Luck, S. J. (2007). Do the contents of visual working memory automatically influence attentional selection during visual search? Journal of Experimental Psychology: Human Perception and Performance, 33, 363-377. doi:10.1037/0096-1523.33.2.363 